\title{
Investigation of cardiovascular protective effect of Shenmai injection by network pharmacology and pharmacological evaluation
}

Lin $\mathrm{Li}^{1,2+}$, Dongli Yang ${ }^{1,2+}$, Jinghao $\mathrm{Li}^{1,2}, \mathrm{Lu} \mathrm{Niu}^{1,2}, \mathrm{Ye}$ Chen ${ }^{1,2}$, Xin Zhao ${ }^{1,2}$, Patrick Kwabena Oduro ${ }^{1,2}$, Chun Wei ${ }^{3}$, Zongpei $\mathrm{Xu}^{2}$, Qilong Wang ${ }^{1,2^{*}}$ and Yuhong $\mathrm{Li}^{1,2^{*}}$

\begin{abstract}
Background: Shenmai injection (SMI) has been used in the treatment of cardiovascular disease (CVD), such as heart failure, myocardial ischemia and coronary heart disease. It has been found to have efficacy on doxorubicin (DOX)induced cardiomyopathy. The aims of this study were to explore the underlying molecular mechanisms of SMI treatment on CVD by using network pharmacology and its protective effect on DOX-induced cardiotoxicity by in vitro and in vivo experiment based on network pharmacology prediction.

Methods: Network pharmacology method was used to reveal the relationship between ingredient-target-disease and function-pathway of SMI on the treatment of CVD. Chemical ingredients of SMI were collected form TCMSP, BATMAN-TCM and HIT Database. Drugbank, DisGeNET and OMIM Database were used to obtain potential targets for CVD. Networks were visualized utilizing Cytoscape software, and the enrichment analysis was performed using IPA system. Finally, cardioprotective effects and predictive mechanism confirmation of SMI were investigated in H9c2 rat cardiomyocytes and DOX-injured C57BL/6 mice.

Results: An ingredient-target-disease \& function-pathway network demonstrated that 28 ingredients derived from SMI modulated 132 common targets shared by SMI and CVD. The analysis of diseases \& functions, top pathways and upstream regulators indicated that the cardioprotective effects of SMI might be associated with 28 potential ingredients, which regulated the 132 targets in cardiovascular disease through regulation of $\mathrm{G}$ protein-coupled receptor signaling. In DOX-injured H9c2 cardiomyocytes, SMI increased cardiomyocytes viability, prevented cell apoptosis and increased PI3K and p-Akt expression. This protective effect was markedly weakened by PI3K inhibitor LY294002. In DOX-treated mice, SMI treatment improved cardiac function, including enhancement of ejection fraction and fractional shortening.
\end{abstract}

Conclusions: Collectively, the protective effects of SMI on DOX-induced cardiotoxicity are possibly related to the activation of the PI3K/Akt pathway, as the downstream of G protein-coupled receptor signaling pathway.

Keywords: Network pharmacology, Shenmai injection, Doxorubicin, Cardiotoxicity, PI3K/Akt signaling pathway

\footnotetext{
*Correspondence: wangqilong_00@tjutcm.edu.cn; liyuhong@tjutcm.edu.cn

${ }^{\dagger}$ Lin Li and Dongli Yang contributed equally to this work.

${ }^{1}$ Institute of Traditional Chinese Medicine, Tianjin University of Traditional

Chinese Medicine, Tianjin 301617, China

Full list of author information is available at the end of the article
} 


\section{Background}

Doxorubicin (DOX) is a widely used chemotherapeutic drug in the treatment of human solid and hematogenous malignancies. However, it has cumulative dosedependent cardiotoxic effects, which can lead to cardiac dysfunction, cardiomyopathy, and even severe heart failure [1]. When the cumulative dose of DOX is $400 \mathrm{mg} /$ $\mathrm{m}^{2}$, the risk of heart failure is at an average of $5 \%$. This risk increased exponentially at higher doses of DOX [2]. Moreover, the incidence of subclinical and overt cardiotoxicity in cancer patients treated with DOX after 9 years follow-up was 17.9 and 6.3\%, respectively [3]. Therefore, with the increasing population of cancer survivors treated with DOX, its cardiotoxicity arises a wide concern. Dexrazoxane (DEX) is an established cardioprotectant, with a protective effect of the heart from DOX. DEX, the only Food and Drug Administration (FDA) approved-medicine that is used in combination with DOX. However, evidence showed that DEX aggravates bone marrow suppression induced by chemotherapeutic drugs [4]. Therefore, it is urgent to find cardioprotective medicines with both high efficiency and low toxicity as the combined medication with chemotherapeutic drugs.

Traditional Chinese Medicine (TCM), which featured as having "multiple ingredients and multiple targets", has been used for treating complex diseases for decades [5]. Shenmai injection (SMI), composed of Ginseng Radix et Rhizoma Rubra (GR) and Ophiopogonis Radix $(\mathrm{OR})$, is derived from Shengmaisan in Qianjin Yaofang. SMI has been approved by the China Food and Drug Administration (CFDA) for the treatment of chronic corpulmonale heart failure since 1995 [6]. Shreds of evidence have reported that the mechanisms of SMI in the treatment of CVD were related to improving the electrophysiological activity in hypertrophic rat myocardium [7], up-regulating nitric oxide level, increasing superoxide dismutase activity, decreasing endothelin-1 levels,

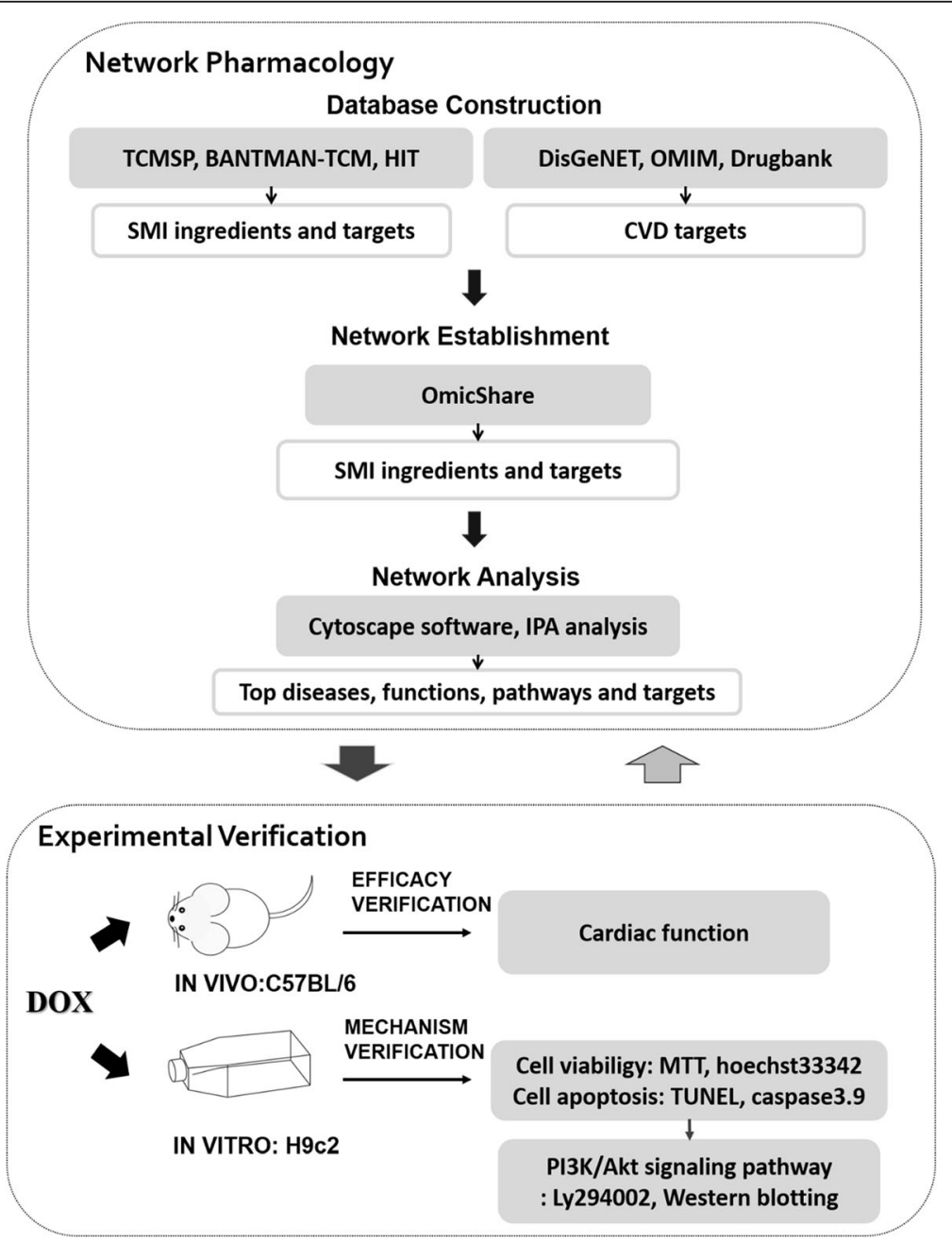

Fig. 1 Workflow of the entire research 
and improving vascular endothelial-dependent vasodilation [8]. However, the underlying molecular mechanisms of the cardioprotective effect of SMI remain unexplored.

In 1999, SMI was first reported to be used in the treatment of DOX-induced cardiotoxicity [9]. Recent evidence showed that SMI prevented abnormal electrocardiogram, left ventricular ejection fraction (LVEF), and cardiac troponin (cTnT) caused by DOX [10]. Moreover, SMI can even reduce the incidence of bone marrow suppression caused by DEX $[11,12]$. Although studies have reported that the protective effect of SMI on DOXinduced myocardial damage may be associated with scavenging free radical [13], relieving calcium overload [14], and protecting mitochondria function [15], the underlying molecular mechanisms has not been elucidated.

Network pharmacology has helped to unveil the complicated pharmacological mechanism of several TCM formulations by combining cheminformatics, bioinformatics, and network biology [16, 17]. SMI is a multi-component and multi-target agent, which exhibits cardioprotective efficacy through regulating molecular networks. Therefore, in this research, network pharmacology and experimental verification were combined to elucidate the potential mechanism of SMI on DOXinduced cardiotoxicity (Fig. 1). Further, H9c2 cells were used in vitro for mechanism verification. C57BL/6 mice injured by DOX were used in vitro for confirming cardioprotective efficacy of SMI.

\section{Methods \\ SMI ingredients collection and targets fishing}

The chemical ingredients of GR and OR were collected from the Traditional Chinese Medicine Systems Pharmacology (TCMSP) Database [18] (http://lsp.nwu.edu.cn/ tcmsp.php) and the Bioinformatics Analysis Tool for Molecular mechANism of Traditional Chinese Medicine (BATMAN-TCM) Database [19] (http://bionet.ncpsb. org/batman-tcm/). Drug-likeness (DL) value, as a suggested criterion by TCMSP Database, was used for evaluation of absorption, distribution, metabolism and excretion (ADME) [20]. The ingredients were screened by $\mathrm{DL}$ value. When the $\mathrm{DL}$ value is $\geq 0.18$, the ingredients were retained. Literature mining also supplemented the information from these databases. The protein targets of compounds from SMI were mainly collected from TCMSP and BATMAN-TCM. As a supplement, the Herbal Ingredients' Targets (HIT) Database [21] (http://lifecenter.sgst.cn/hit/) was used for obtaining the validated targets. Due to the irregular naming of the retrieved target, UniProt Database [22] (http://www.uni prot.org/) was used for correcting all retrieved proteins to their official symbols by inputting protein names and restricting species to human beings.

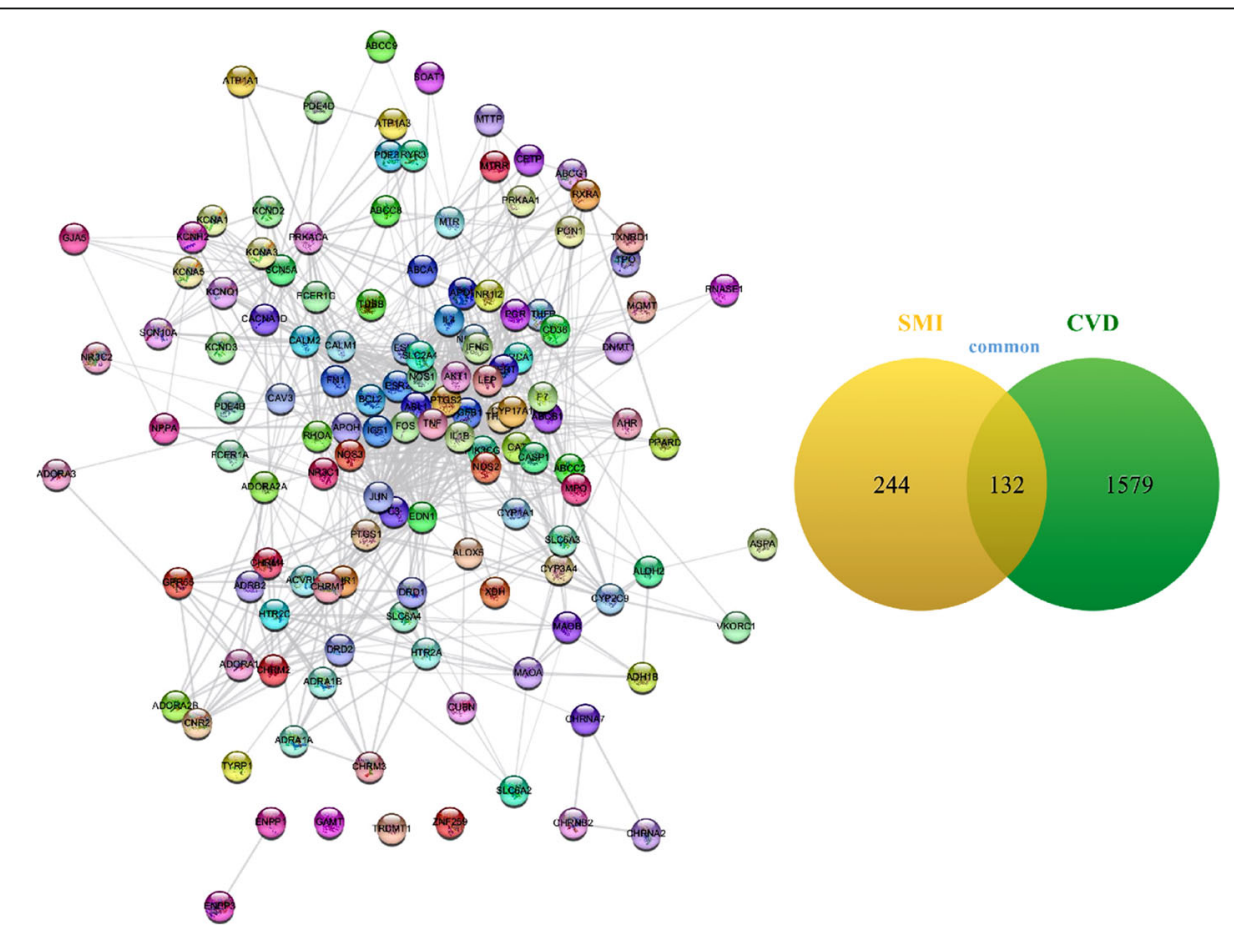

Fig. 2 Identification of common targets of CVD and SMI. Common targets of CVD and SMI PPI network. Edges represent protein (node) interactions. Line thickness indicates the strength of data support. Venn diagrams (right) demonstrate the number of shared and unique targets by SMI and CVD 


\section{Cardiovascular disease genes collection}

The potential targets for CVD were obtained from three resources: Drugbank Database [23] (https://www.drug bank.ca/), a database of gene-disease associations (DisGeNET) [24] (http://www.disgenet.org/), and Online Mendelian Inheritance in Man (OMIM) [25] (http:// omim.org/).

\section{Identification of common targets of CVD and SMI}

The OmicShare tool (http://www.omicshare.com/tools) was used for identification of the common targets of CVD and SMI. Briefly, the SMI and CVD associated targets dataset was constructed and uploaded into the Venn tool to identify common targets of CVD and SMI.

\section{Network construction and analysis}

All the networks were visualized utilizing Cytoscape software [26] (Version 3.6.0). Network construction was performed as follows: (1) SMI targets PPI network; (2) CVD targets PPI network; (3) common targets of CVD and SMI PPI network; (4) SMI ingredient-target network; (5) target-disease \& function-pathway network.

Enrichment Analysis was performed by the Ingenuity Pathway Analysis (IPA, version 01-12) system [27]. "Core analysis" module was used for obtaining top canonical pathways, upstream regulators, diseases and bio functions. The concerned canonical pathways were found by using "Overlay-Canonical Pathway" module. Then "Build-Diseases \& Functions" module was performed to obtain the targets involved in diseases and bio functions. "Path designer" module was used for prettifying the network. The $P$-values represent the possibility that the molecules in the dataset were related to canonical pathways/diseases and bio functions/upstream regulators. The enrichment score of P-values was based on Fisher's exact test.

\section{Cell culture and treatments}

Rat embryonic ventricular myocardial cell line H9c2 was purchased from the American Type Cell Culture (ATCC, Manassas, VA). Cells were cultured as

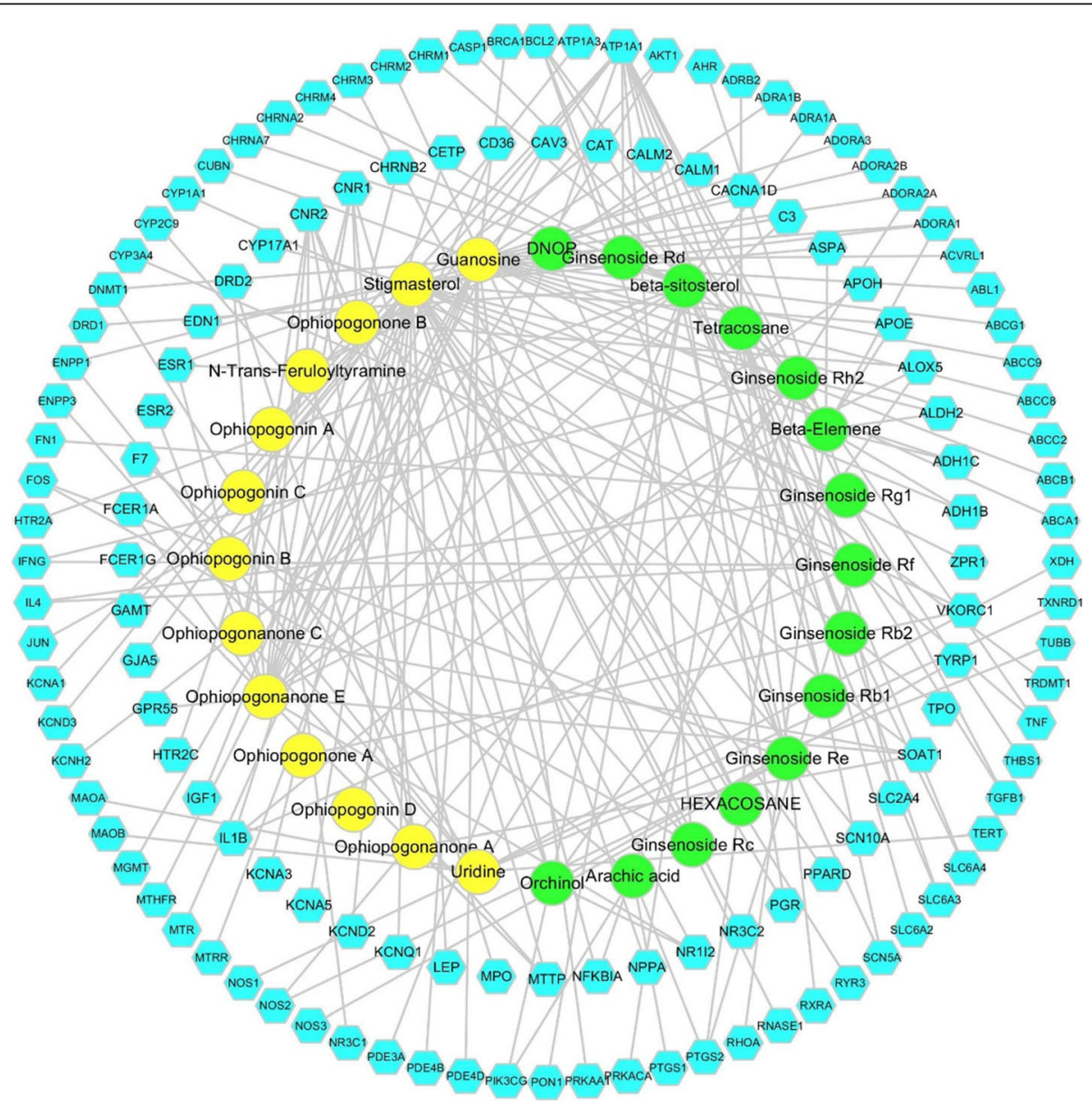

Fig. 3 Establishment of ingredient-target network of SMI. Fifteen GR ingredients (green) and thirteen OR ingredients (yellow) cooperatively modulated the 132 common targets (light blue) 
previously described [28]. Cells were pretreated with or without SMI $(0.25 \%$ or $0.5 \%$ in DMEM) for $24 \mathrm{~h}$. The pretreated cells were then treated with or without DOX (Sigma, St. Louis, MO, USA, $0.5 \mu \mathrm{M}$ ) for $24 \mathrm{~h}$. Moreover, in some group, LY294002 (Selleckchem, USA, $10 \mu \mathrm{M}$ ) was added to cells for $1 \mathrm{~h}$ before DOX treatment.

\section{Cell viability measurements}

Cell viability was examined by a cell counting kit 8 (CCK8, Dojindo Laboratories, Japan). After H9c2 cells were treated with DOX in the absence or presence of SMI or LY294002 at $37^{\circ} \mathrm{C}$ in a 96 -well plate, CCK8 solution $(10 \mu \mathrm{L} / 100 \mu \mathrm{L}$ medium) was added to the media for $3 \mathrm{~h}$. The absorbance of the media at $450 \mathrm{~nm}$ was examined by a microplate reader (Infinitef50, Tecan, Switzerland).

\section{Nuclear staining with Hoechst 33342}

Cells were incubated with DOX in the absence or presence of SMI or LY294002 at $37^{\circ} \mathrm{C}$. Then Hoechst 33342 (Sigma, St. Louis, MO, USA, $10 \mu \mathrm{g} / \mathrm{mL}$ ) was applied for 15 min under dark condition to stain the nucleus of cells. Images were observed, and photomicrographs were taken with a fluorescence microscope (AX10, Carl Zeiss AG, Germany). Cells with a nucleus exhibiting brightly stained condensed chromatin, unclear fragments or apoptosis bodies were identified as damaged nucleus. The positive staining nucleus was calculated in 3 random fields $(\times 630)$. The damaged nucleus index was presented as the percentage of the number of positively stained nuclei to the number of all stained nuclei from 3 random fields in each group.

\section{TUNEL staining}

The staining assay was measured using TUNEL kit (Roche, Mannheim, Germany). Apoptotic cells were counted in 3 random fields $(\times 100)$ by fluorescence microscope in each group.

\section{Western blotting}

Total protein obtained from cardiac tissue and cardiomyocytes were quantified using bicinchoninic acid protein assay kits (CWBIO, Jiangsu, China). Equal amounts of proteins were separated by NUPAGE ${ }^{\mathrm{Tm}} 10 \%$ Bis-Tris Gels and transferred onto a polyvinylidene fluoride (PVDF) membrane (Immobilon, USA). After being blocked, the membranes were incubated overnight at $4{ }^{\circ} \mathrm{C}$ with the corresponding primary antibody. After washing with TBST, the membranes were incubated for $1.5 \mathrm{~h}$ with secondary antibodies. All antibodies were purchased from Cell Signaling Technology (Danvers, MA, USA). The immunoblots were detected by western

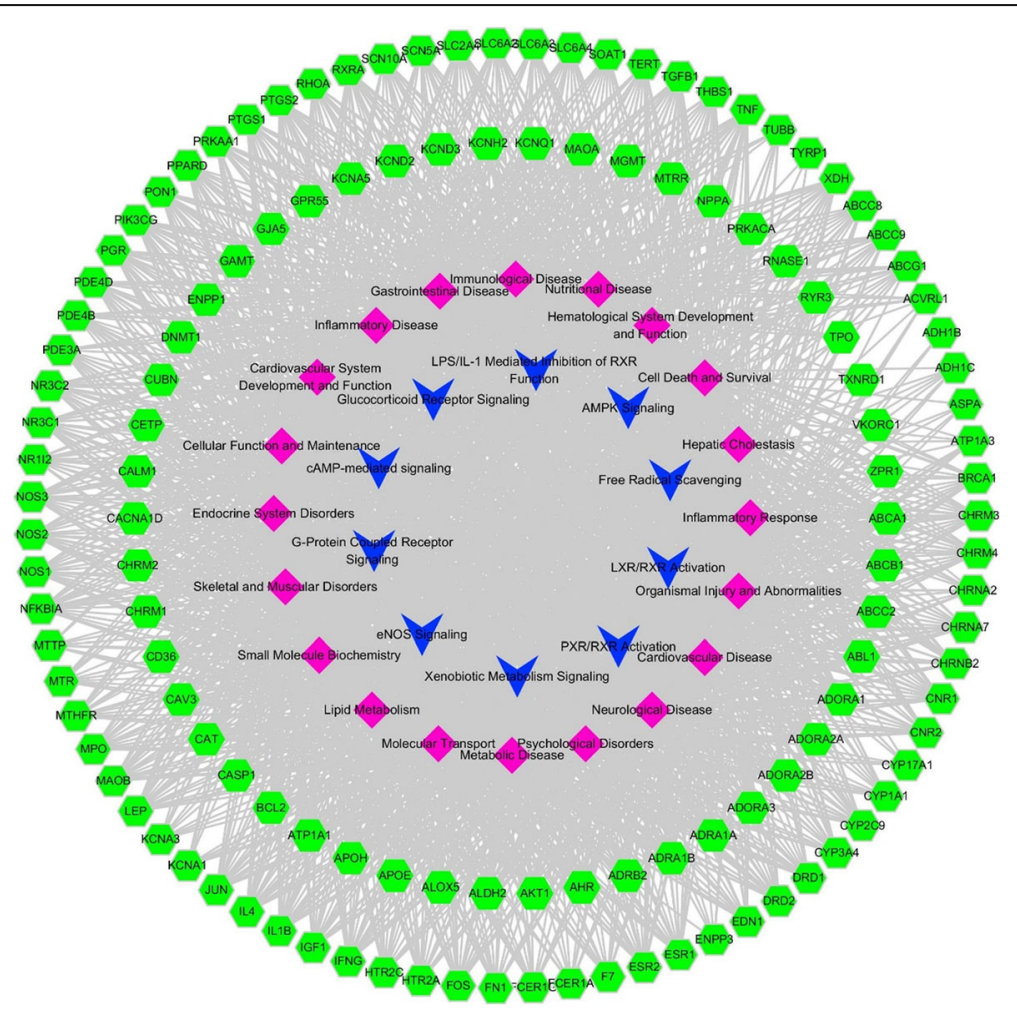

Fig. 4 Establishment of target-disease \& function-pathway network of SMI. Top 10 canonical pathways (blue), top 20 diseases and bio functions (pink) correlative with the 129 common targets (green) were shown 
chemiluminescent HRP substrate (Immobilon, USA) and Amersham Imager 600 (GE Healthcare Bio-Sciences AB, Japan).

\section{Animal study}

Male C57BL/6 mice weighing 22-25 g were purchased from Beijing Vital River Laboratory Animal Technology Co., Ltd. (certificate number: SCXK(JING)20,160,006). All animal experiments were carried out according to the National Institute of Health Guide for the Care and
Use of Laboratory Animals, and were approved by the Animal Care and Use Committee of Tianjin University of Traditional Chinese Medicine. Mice were given free access to water and normal food under a 12-h light-dark cycle and specific pathogen-free conditions.

DOX (Shenzhen Main Luck Pharmaceuticals, Shenzhen, China) was dissolved in normal saline. Mice were randomly divided into 4 groups: normal control group (CON, $n=10)$, SMI control group (CON+SMI, $\mathrm{n}=10$ ), DOX injury group (DOX, $n=26$ ) and SMI

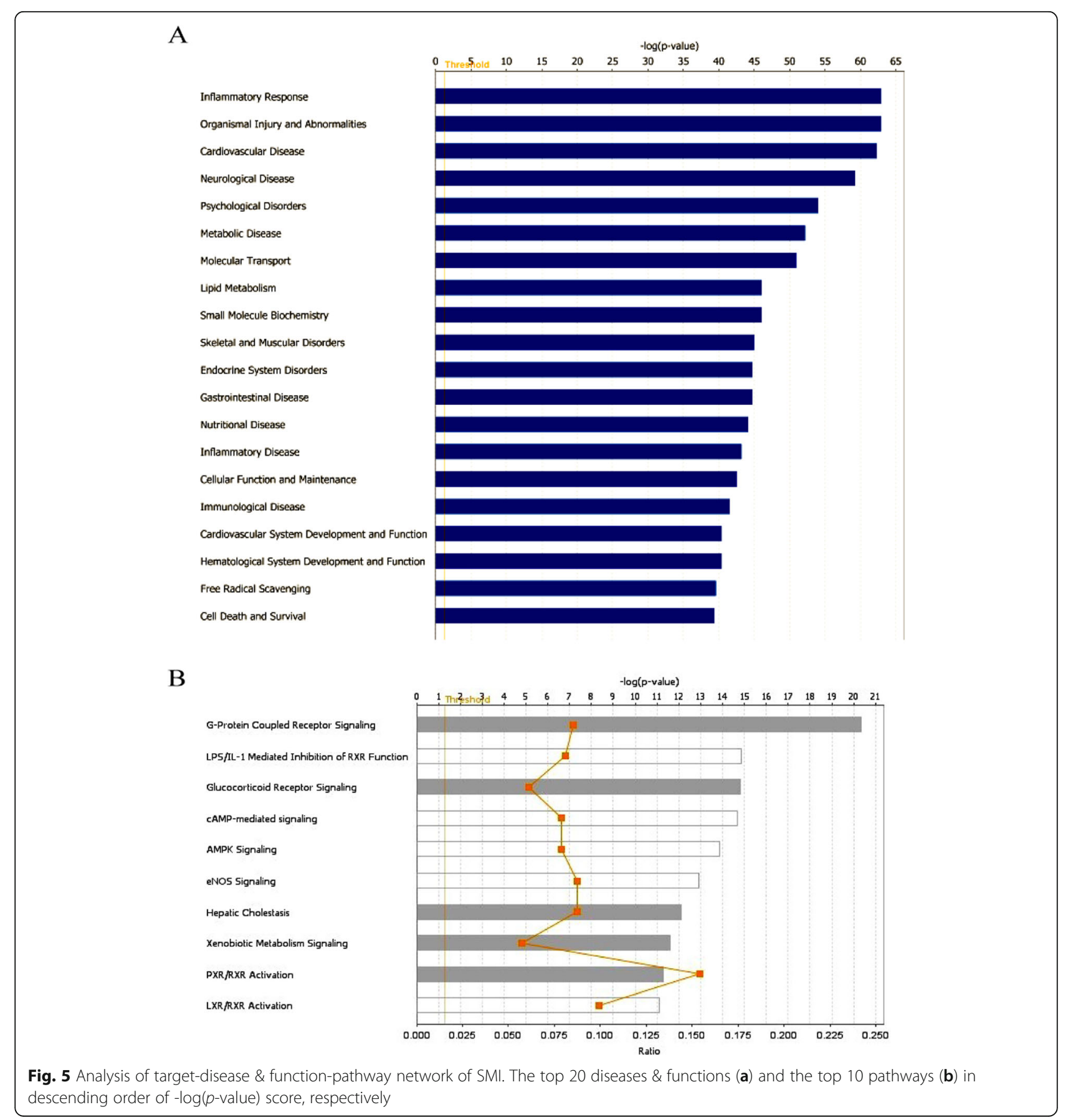


pretreatment group (DOX + SMI, $n=25)$. Due to the possibility of DOX-induced death, the number of mice in DOX injured-group was higher than the normal group, i.e., $\mathrm{n}=26$ and $\mathrm{n}=10$, respectively. Mice received a daily intraperitoneal (i.p.) injection of DOX (3 mg/kg/day for 5 days, i.p.) to obtain a cumulative dose of $15 \mathrm{mg} / \mathrm{kg}$. SMI (Chia Tai Qingchunbao Pharmaceutical, Hangzhou, China, $2.5 \mathrm{ml} / \mathrm{kg} /$ day for 8 days, i.p.) was given 3 days before administration of DOX. Control mice received an intraperitoneal injection of normal saline. After $24 \mathrm{~h}$ of the last dose of DOX, body weight was recorded, and left ventricular function was evaluated. At the end of the experiment, all animals were anaesthetized with tribromoethanol solution ( $\mathrm{g}$ 2,2,2-tribromoethanol dissolved in $0.625 \mathrm{~mL}$ 2-methyl-2-butanol and diluted to $20 \mathrm{mg} / \mathrm{mL}$ with normal saline, $0.1 \mathrm{~mL} / 10 \mathrm{~g}$ body weight, i.p.). When mice were unconscious, blood was sampled from their orbital vein, and after which, they were euthanized by cervical dislocation, for immediate heart collection. Heart weight and tibial length were measured.

\section{Echocardiographic assessment}

Echocardiography was performed using a Vevo 2100 Imaging System (Visual Sonics, Toronto, Canada). The mice were inhaled with isoflurane (1\% oxygen plus $2-5 \%$ isoflurane) to induce anesthesia. Two-dimensional image obtained in the parasternal long-axis views. The percentage of fractional shortening (FS, \%), ejection fraction $(E F, \%)$ and left ventricular posterior wall thickness (LVPW) were determined from the M-mode recordings.

\section{Assessment of serum markers of cardiac injury}

Creatine kinase $(\mathrm{CK})$, creatine kinase-MB isoenzyme (CK-MB) and lactate dehydrogenase (LDH) activities were measured using commercial kits (BIOSINO, Beijing, China) and automatic biochemical analyzer (Thermo Fisher Scientific, NY, USA).

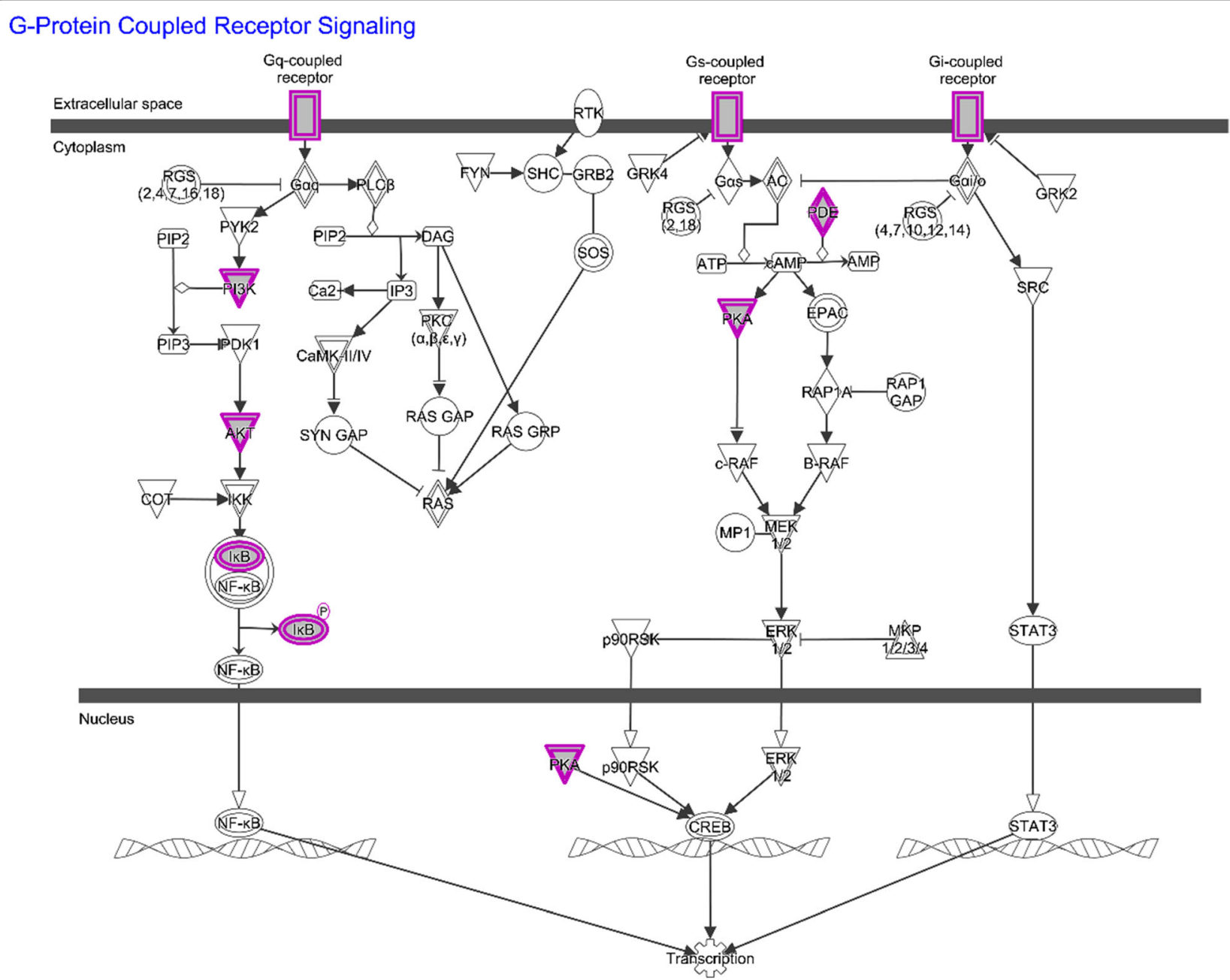

Fig. 6 Detailed signaling pathway of G-protein coupled receptor. Targets identified from the 132 common targets were highlighted in purple 


\section{Statistical analysis}

Data were expressed as mean \pm SD. Statistical evaluations were performed by one-way analysis of variance (ANOVA) followed by LSD Method and Dunnett's C test, using SPSS software (Version 24.0). Values of $P<$ 0.05 were considered a statistically significant difference.

\section{Results}

\section{Identification of common targets of CVD and SMI}

Through mining the TCMSP, BATMAN-TCM, HIT, Drugbank, DisGeNET and OMIM Database, a total of 376 targets related to SMI (Figure S1) and 1711 targets related to CVD (Figure S2) were obtained. Among them, 132 targets were shared by both SMI and CVD (Fig. 2), which were listed in Table 1 (supplementary data), and became the focus of our following analysis.

\section{Establishment and analysis of Ingredient-Target-Disease \& Function-Pathway Network}

In order to explain potential pharmacological effects of SMI on the treatment of CVD, compound-target and target-disease \& function-pathway networks were performed. SMI ingredient-multiple target network consisted of 160 nodes and 198 edges, which elucidated the 28 ingredients of SMI (15 and 13 derived from GR and OR, respectively) and their modulated 132 common targets. The interactions indicated that one compound could regulate numerous targets, such as beta-sitosterol, ginsenoside Rf, ginsenoside Rg1, ginsenoside Rh2, ginsenoside $\mathrm{Rb} 1$, ginsenoside $\mathrm{Rd}$., guanosine, ophiopogonin $\mathrm{D}$, ophiopogonanone E, stigmasterol and so on. In the meanwhile, a single target may be regulated by multiple compounds like ATP1A1, BCL2, CNR1, CNR2, SOAT1, MTTP, AKT1, NOS2, et al. (Fig. 3). Combined with the core analysis results of IPA, a target-disease \& functionpathway network was constructed by Cytoscape. This network consisted of 159 nodes (10 pathways, 20 diseases \& functions, 129 targets) and 2068 edges, which illustrated the biological process and molecular mechanisms of the common targets (Fig. 4).

The core analysis of diseases \& bio functions and canonical pathways were ranked according to $p$-value score. The top 20 diseases \& bio functions and top 10 canonical pathways were showed respectively. The top 20 diseases \& bio functions by SMI, in descending order of $-\log (p$-value) score, were inflammatory response,

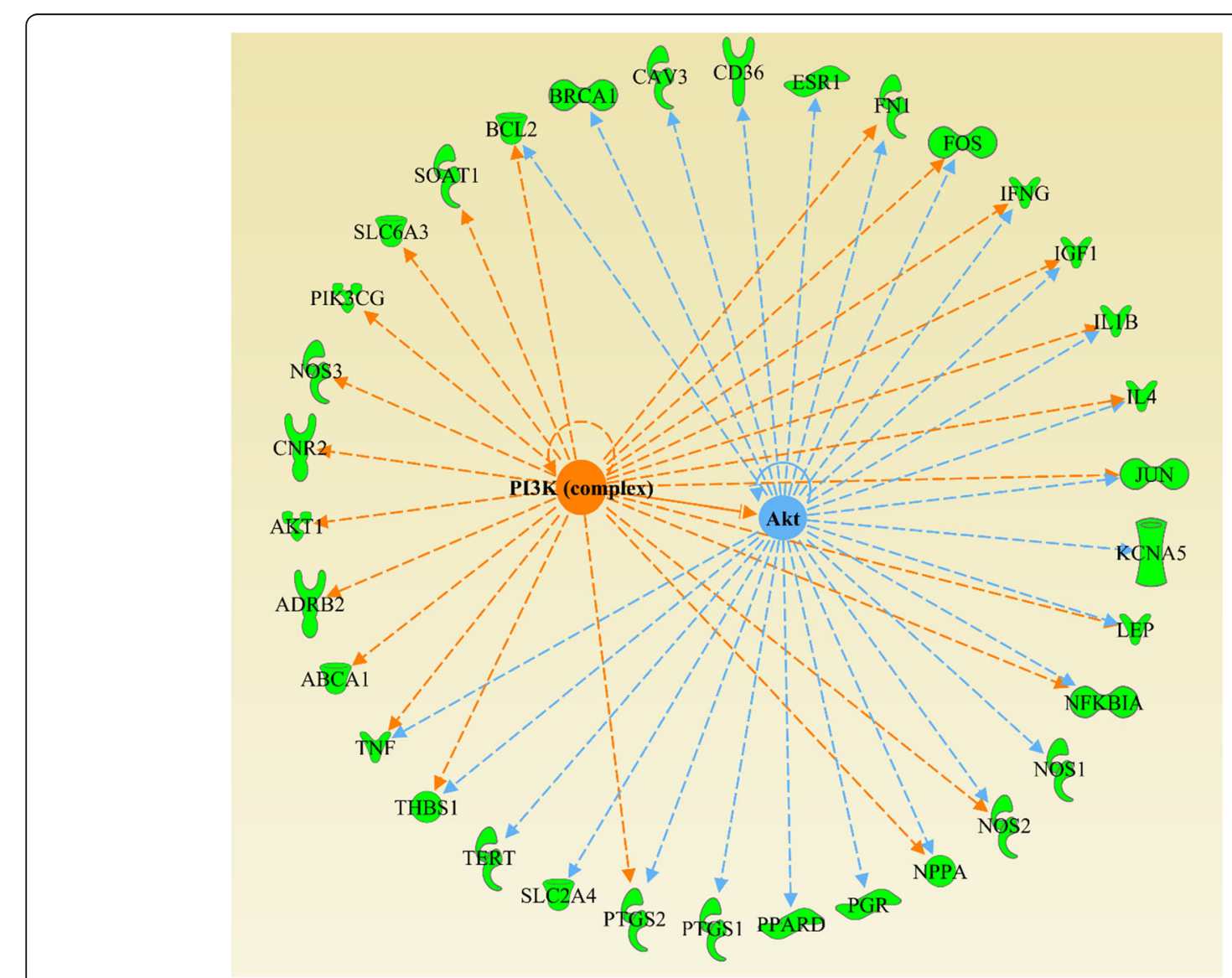

Fig. 7 The relationship of PI3K and Akt including their downstream regulated targets 
organismal injury and abnormalities, cardiovascular disease, neurological disease, psychological disorders, metabolic disease, molecular transport, lipid metabolism, small molecule biochemistry, skeletal and muscular disorders, endocrine system disorders, gastrointestinal disease, nutritional disease, inflammatory disease, cellular function and maintenance, immunological disease, cardiovascular system development and function, hematological system development and function, free radical scavenging, cell death and survival. Among them, cardiovascular disease was the third highest one (Fig. 5a). Analysis of IPA canonical pathways showed that the top 10 canonical pathways in descending order of $-\log (p$ value) score were G-protein coupled receptor signaling, LPS/IL-1 mediated inhibition of RXR function, glucocorticoid receptor signaling, cAMP-mediated signaling, AMPK signaling, eNOS signaling, hepatic cholestasis, xenobiotic metabolism signaling, PXR/RXR activation and LXR/RXR activation. The G-protein coupled receptor signaling was considered as the essential pathway with the $-\log$ (p-value) score of 20.3 (Fig. 5b, Table 2 in supplementary data). The detailed pathways of the Gprotein coupled receptor signaling, including the identified molecular targets, were presented in Fig. 6.

The top 20 upstream regulators and their corresponding targets of the 132 common targets were obtained from "Core analysis" (Table 3 in supplementary data).
Among them, PI3K and Akt were shown to have a protein-protein interaction (Fig. 7), which were also the primary targets in the G-protein coupled receptor signaling.

Collectively, the results imply that PI3K-Akt signaling pathway, as the downstream in the $\mathrm{G}$ protein-coupled receptor signaling, may serve as CVD-related mechanism for SMI. It has been reported that PI3K/Akt signaling pathway has a direct correlation with DOX-induced cardiotoxicity $[29,30]$. Although studies have reported protective effects of SMI on DOX-induced myocardial damage, the underlying molecular mechanisms remain unclear [13-15]. Accordingly, we further explored the cardioprotective mechanism of SMI on DOX-induced cardiomyocyte injury.

\section{Protective effects of SMI on DOX-induced cell death and apoptosis}

To assess the effect of SMI on DOX-induced cardiotoxicity, we first measured the effects of different concentrations of DOX on the viability of $\mathrm{H} 9 \mathrm{c} 2$ cells by CCK8 assay. As shown in Fig. 8a, DOX caused a decrease in the cell viability, when its concentration exceeded $0.25 \mu \mathrm{M}$ for $24 \mathrm{~h}(P<0.01)$. DOX of $0.5-$ $10 \mu \mathrm{M}$ induced a stable and significant reduction of cell viability. Based on these, a concentration of $0.5 \mu \mathrm{M}$ was selected and used for subsequent
A

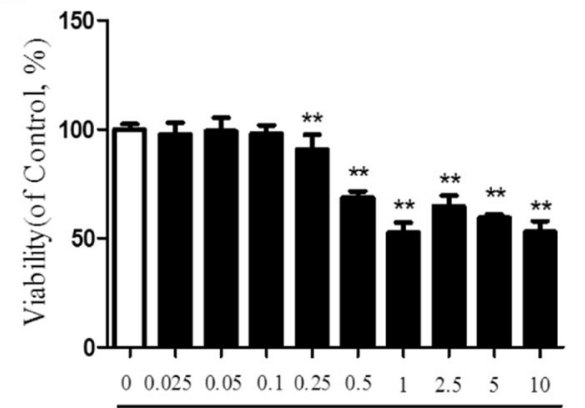

$\operatorname{DOX}(\mu \mathrm{M})$

$\mathrm{C}$

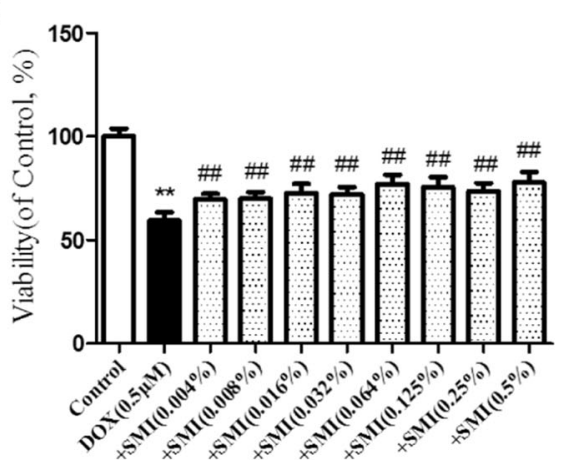

B

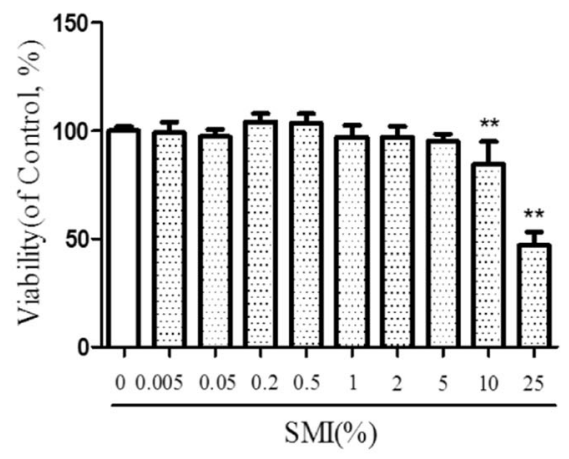

Fig. 8 Effects of SMI on DOX-impaired cell viability of H9c2 cells. a Toxic effect of DOX on cell viability. $\mathbf{b}$ Toxic effect of SMI on cell viability. $\mathbf{c}$ Effects of SMI on the viability of DOX-induced H9c2 cells. ${ }^{* *} P<0.01$ vs. Control; ${ }^{\#} P<0.01$ vs. DOX 
experiment. The cell viability was significantly lower in the high concentration of SMI treatment group $(10,25 \%)$ than in the control group $(P<0.01)$; however, no significant difference appeared between the low concentration of SMI $(0.005,0.05,0.2,0.5,1,2$, $5 \%)$ and the control group $(P>0.05$, Fig. 8b). In addition, compared with the cells in DOX $(0.5 \mu \mathrm{M}$, $24 \mathrm{~h})$ - injured group, treatment with SMI (0.004, $0.008,0.016,0.032,0.064,0.125,0.25,0.5 \%)$ significantly increased cell viability $(P<0.01$, Fig. $8 \mathrm{c})$.

Hoechst33342 staining assay showed that DOXinduced damaged nuclei, as demonstrated by condensed chromatin, unclear fragments, or apoptosis bodies. However, pre-treatment with SMI (0.5\%) significantly decreased the number of damaged nucleus $(P<0.01$, Fig. 9a). Using TUNEL assay, DNA fragmentation was measured. DOX triggered an increase in TUNEL-positive cells, which were significantly reduced when pretreated with SMI $(P<0.01$, Fig. 9b).

The activation of caspase- 9 and caspase- 3 participates in the apoptosis of mitochondrial-dependent pathway. The relative protein levels of cleaved caspase- 9 and cleaved caspase- 3 were significantly elevated in DOX-treated cells when compared with the control cells $(P<0.01$, Fig. 9c). However, this effect was significantly inhibited by SMI pretreatment $(P<$ 0.01, Fig. 9c).

\section{A}
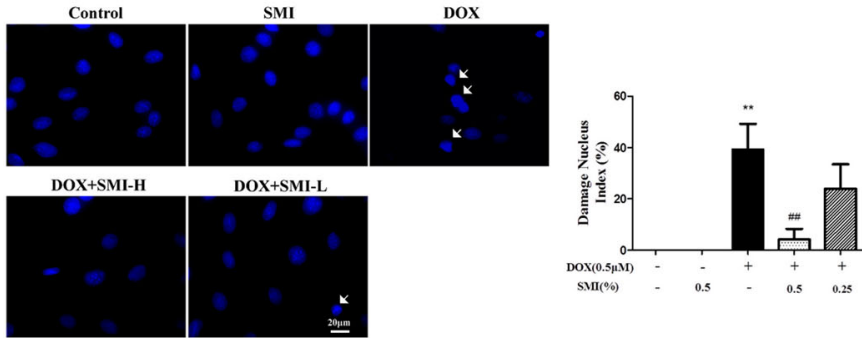

B
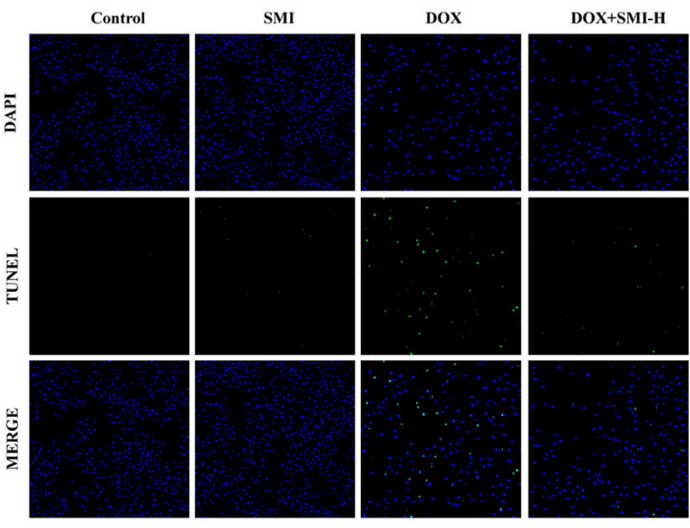

DOX+SMI-L
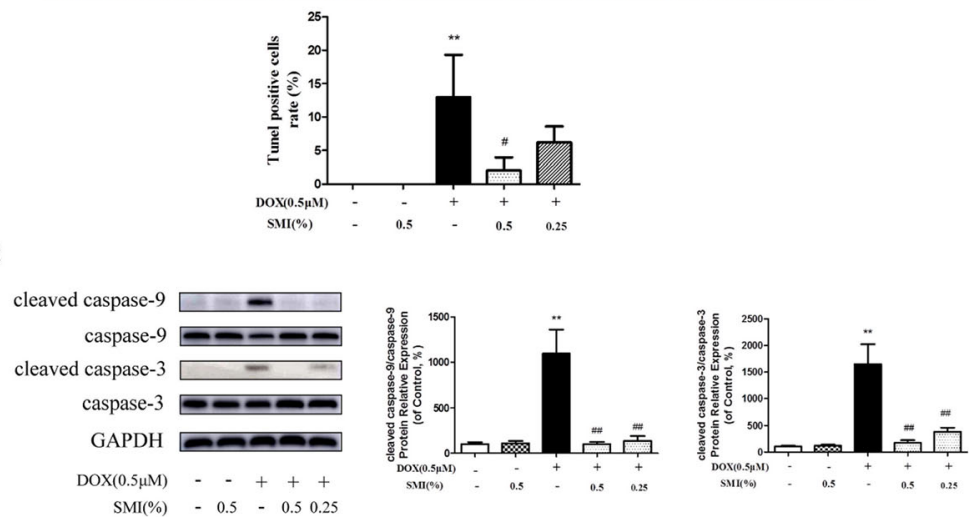

Fig. 9 Effects of SMI on DOX-induced apoptosis in H9c2 cells. Cells were pretreated with SMI $(0.25,0.5 \%)$ for $24 \mathrm{~h}$, then stimulated with DOX $(0.5 \mu \mathrm{M})$ for $24 \mathrm{~h}$. a Damaged nucleus was examined by Hoechst 33342 staining. Damaged nuclei are marked with white arrows. b Cell apoptosis was assessed by TUNEL assay. c Protein levels of cleaved caspase-9, caspase-9, cleaved caspase-3 and caspase-3 were measured by Western blot. ${ }^{* *} P<0.01$ vs. Control; ${ }^{\#} P<0.05$ vs. DOX; ${ }^{\# \#} P<0.01$ vs. DOX 
Effects of SMI on DOX-induced apoptosis through modulation of the PI3K/Akt signaling pathway

To determine the impact of SMI on DOX-induced cardiomyocyte injury, we examined the protein expression of PI3K and p-Akt in H9c2 cells. As depicted in Fig. 10a, the protein levels of PI3K and phosphorylation of Akt were significantly diminished in DOX-treated cells, when compared with the control cells $(P<0.01, P<0.05)$. However, pretreatment of SMI increased the PI3K and p-Akt expression, compared with the DOX group $(P<0.05)$.

Moreover, PI3K inhibitor LY294002 blocks the anti-apoptosis effect of SMI, with reduced expression of PI3K and p-Akt (Fig. 10b), increased number of damaged nuclei (Fig. 11a) and apoptosis cells (Fig. 11b) in the SMI pretreatment plus DOX group. As shown in Fig. 11c, DOX dramatically elevated the relative protein levels of cleaved caspase-9 and cleaved caspase-3 $(P<0.01)$, which was markedly suppressed by SMI pretreatment. However, LY294002 significantly inhibited these regulating effects of SMI on caspase-9 and caspase-3 activation $(P<0.01$, Fig. 11c). These results suggested that PI3K/Akt signaling pathway was involved in SMImediated cardiomyocyte protection.

\section{Protective effects of SMI on DOX-induced Cardiotoxicity} in mice

We validated the effect of SMI on improvement of cardiac dysfunction in acutely injured mice induced by DOX. SMI pretreatment mitigated the body weight loss induced by DOX (Fig. 12a). The heart-to-body weight ratio $(\mathrm{HW} / \mathrm{BW})$ were unchanged among all the experimental mice (Fig. 12b). Although CK, CK-MB and LDH levels were not significantly elevated in DOX-treated mice compared with their levels in normal control mice (Fig. 12c-e), echocardiography assessments showed that levels of EF and FS were significantly decreased in DOXtreated mice, which were markedly inhibited by SMI pretreatment (Fig. 12g, h). Together, these results indicated that SMI improved cardiac dysfunction induced by DOX in mice.

\section{Discussion}

In this study, we showed evidence about the molecular mechanism behind SMI cardioprotective action against DOX-mediated cardiotoxicity using network pharmacology tools. Network pharmacology has been applied in foreseeing the pharmacological mechanisms of TCM [31-33]. Although predictable mechanisms could be obtained from published reports, network pharmacology

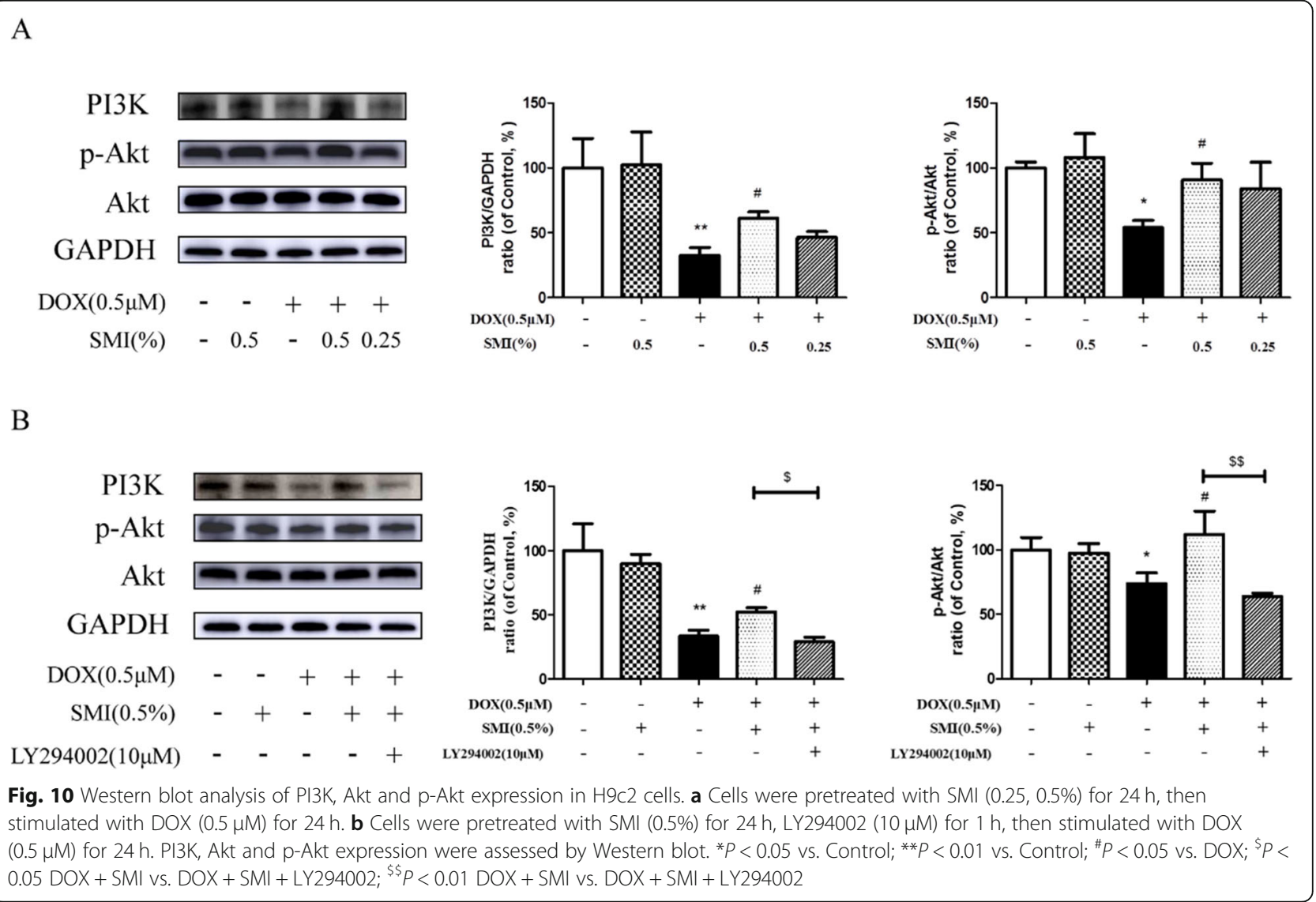



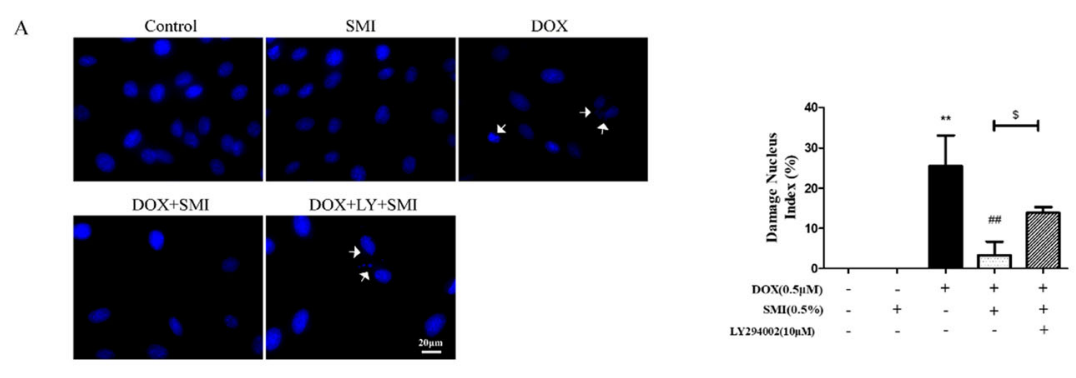

B
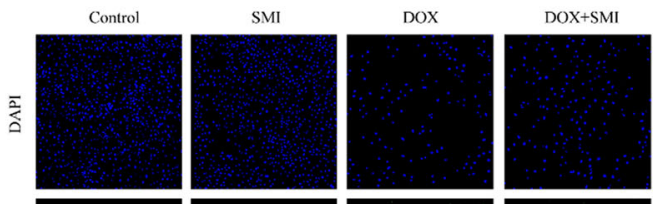

DOX+LY+SMI
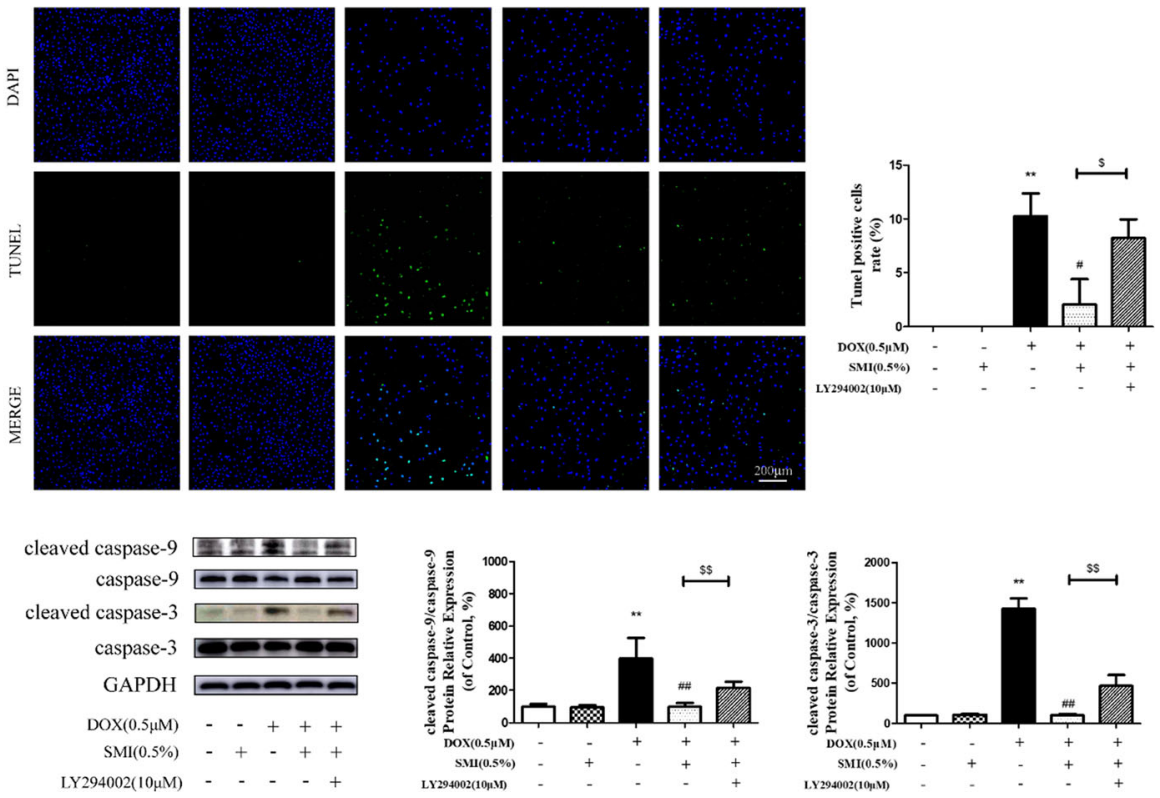

Fig. 11 Effects of LY294002 on the protection of SMI on DOX-induced apoptosis in H9c2 cells. Cells were pretreated with SMI (0.5\%) for $24 \mathrm{~h}$, LY294002 $(10 \mu \mathrm{M})$ for $1 \mathrm{~h}$, then stimulated with DOX $(0.5 \mu \mathrm{M})$ for $24 \mathrm{~h}$. a Damaged nucleus was determined by Hoechst 33342 staining. Damaged nuclei are marked with white arrows. b Cell apoptosis was determined by TUNEL assay. c Protein levels of cleaved caspase-9, caspase-9, cleaved caspase-3 and caspase-3 were assessed by Western blot. ${ }^{* *} P<0.01$ vs. Control; ${ }^{\# \#} P<0.01$ vs. DOX; ${ }^{\$} P<0.05$ DOX + SMI vs. DOX + SMI + LY294002; ${ }^{\$ \$} P<0.01 \mathrm{DOX}+\mathrm{SMl}$ vs. DOX + SMl + LY294002

helps to elucidate the action mechanism of TCM at molecular level with a systematic viewpoint [34]. Therefore, it has been a promising holistic strategy for TCM research. In the present study, network pharmacology study suggested that 28 potential ingredients in SMI may play core roles in regulating the 132 targets, which are major related to CVD. In the target-disease \& function-pathway network, the G-protein coupled receptor signaling is an essential pathway. Notably, PI3K and Akt were ranked on the top upstream regulators, which were also the key proteins in G-protein coupled receptor signaling. These suggested that SMI may play a role in the treatment of CVD through the regulation of PI3K/ Akt signaling pathway. Even some reports have already revealed that PI3K/Akt pathway played an important role in CVD. The present research of network pharmacology provided a multi-dimensional research strategy for a complicated TCM formula.
PI3K/Akt signaling pathway is critical for multiple biological processes and provides a vital cell survival signal in cardiomyocytes [35]. Downregulation of Akt is associated with DOX-induced damage and apoptosis in cardiomyocytes [36]. PI3K/Akt activation helps to inhibit DOX-induced cardiomyocytes apoptosis. Moreover, it has been reported that Akt activation improved contractile function of failing hearts [37, 38]. Shreds of evidence have shown that SMI and its ingredients ophiopogonin D activated PI3K/Akt signaling pathway in myocardial ischemia-reperfusion injury [39]. Our previous study suggested that ginsenoside $\operatorname{Rg} 3$ could promote cell viability and attenuate DOX-induced oxidative damage and apoptosis through Akt activation [40]. Ginsenoside Rb1 presents cardioprotective effect against ischemia/reperfusion injury, which involves activating Akt and phosphorylating glycogen synthase kinase $3 \beta$ [41]. 

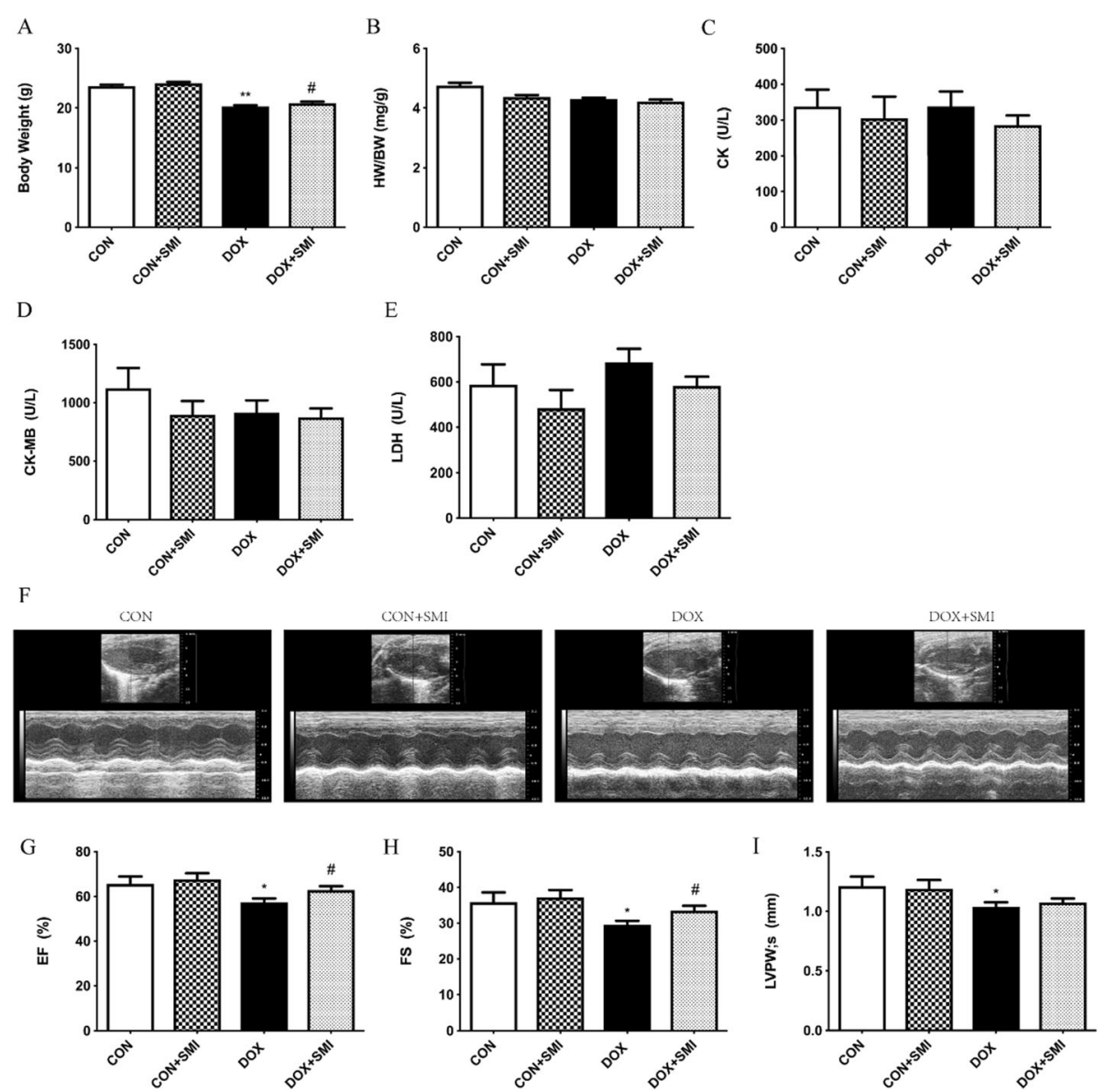

Fig. 12 Protective effects of SMl on DOX-induced cardiotoxicity in mice. a Body weight. $n=10-25$. b Heart weight to body weight ratios (HW/ BW). $n=5-15$. c-e The levels of CK, CK-MB and LDH in mouse serum. $n=7-22$. $\mathbf{f}$ Representative M-mode echocardiograms of mice. $\mathbf{g}$-i Echocardiographic measurement of left ventricular ejection fraction (EF\%), fractional shortening (FS\%), and left ventricular posterior wall (LVPW; s). $n=10-26 .{ }^{*} P<0.05$ vs. Control group; ${ }^{* *} P<0.01$ vs. Control group; ${ }^{\#} P<0.05$ vs. DOX group

Several kinds of research have proved the efficacy of SMI on the treatment of DOX cardiotoxicity in the clinic [10, 11]. Although Shengmai injection, composed of the same two components from SMI and schisandra chinensis, has been reported to be reflective of the energy disruption and cardiac dysfunction induced by DOX [42], the underlying mechanism of SMI on DOXinjured myocardium remains to be explored. Doxinduced cardiotoxicity via activation of apoptosis and suppression of survival pathway. The present study dug deeply into the molecular mechanism of SMI treatment on Dox-induced cardiotoxicity. Accordingly, based on network pharmacology prediction, we focused on the PI3K/Akt signaling pathway for the further experiment of cardioprotective effect of SMI on DOX-induced cardiotoxicity.

We utilized H9c2 cells and acutely injured C57BL/6 mice induced by DOX to explore the protective effect of SMI on the myocardium and verify the prediction results of network pharmacology. In vitro, the pretreatment with SMI prevented loss of cell viability, occurance of cell apoptosis, and damage of nucleus, which were induced by DOX. Moreover, the protein level of PI3K and p-Akt was also downregulated by DOX, while pretreatment with SMI increased the protein level of PI3K and p-Akt. To further verify the regulation of PI3K/Akt signaling pathway to the protective effect of SMI, we also utilized PI3K/Akt inhibitor LY294002. This inhibitor disturbed the protective effect of SMI on DOX-induced cardiotoxicity, which demonstrated a reduction in cell viability, an increase in the number of apoptotic cells, and reduced protein level of PI3K and p-Akt. Also, LY294002 markedly suppressed the SMI-mediated activation of caspase- 9 and caspase- 3 , which are involved in mitochondrial-dependent apoptosis pathway. These findings suggest that SMI protects $\mathrm{H} 9 \mathrm{c} 2$ cardiomyocytes against DOX-induced cytotoxicity, possibly through the activation of the PI3K/Akt pathway.

Additionally, in vivo, we found that pretreatment of SMI rescued decreased body weight and improved left 
ventricular function. Of note, in DOX-treated mice, only LDH levels slightly elevated among cardiac enzymes in serum. A possible explanation for this was that after the last administration of DOX, we evaluated left ventricular function, which resulted in the missing of the window of time for CK, CK-MB and LDH appearance [43, 44]. Therefore, the levels of these cardiac enzymes returned to normal. These findings suggest that SMI exhibited markedly cardioprotective effects on DOX-induced acute injury on mice. Future work will be important to examine the therapeutic effect of simultaneous administration of SMI on the chronic toxicity of DOX.

\section{Conclusions}

In conclusion, network pharmacology analysis suggested that the protective effects of SMI may be associated to its 28 potential ingredients, which regulate 132 targets in CVD through modulation of G-protein coupled receptor signaling. In vitro and in vivo results confirmed that SMI attenuated DOX-induced cardiotoxicity and improved DOX-induced cardiac dysfunction. Furthermore, the protective mechanisms possibly involve the activation of the PI3K/Akt signaling pathway. However, further work will be necessary to verify other signaling pathways and elucidate their relationships.

\section{Supplementary information}

Supplementary information accompanies this paper at https://doi.org/10. 1186/s12906-020-02905-8.

\section{Additional file 1. Supplementary data}

\section{Abbreviations \\ Akt: Protein kinase B; BATMAN-TCM: Bioinformatics Analysis Tool for Molecular mechANism of Traditional Chinese Medicine; CCK8: Cell counting kit 8; CK: Creatine kinase; CK-MB: Creatine kinase-MB isoenzyme; CVD: Cardiovascular disease; CTnT: Cardiac troponin; DEX: Dexrazoxane; DOX: Doxorubicin; EF: Ejection fraction; FDA: Food and drug administration; FS: Fractional shortening; HIT: Herbal Ingredients' Targets; HW/BW: Heart-to- body weight ratio; IPA: Ingenuity Pathway Analysis; LDH: Lactate dehydrogenase; LVEF: Left ventricular ejection fraction; OMIM: Online Mendelian Inheritance in Man; PI3K: Phosphoinositide 3-kinase; SMI: Shenmai injection; TCM: Traditional Chinese Medicne; TCMSP: Traditional Chinese Medicine Systems Pharmacology; TUNEL: Terminal deoxynucleotidyl transferase-mediated dUTP nick end labelling}

\section{Acknowledgements}

Not applicable.

\section{Authors' contributions}

All authors have read and approved the manuscript. QW and $Y L$ designed the study. LL and DY performed the experiments, analyzed the data and drafted the manuscript. $J L, L N$ and $Y C$ helped to perform the experiments. $L L, X Z, C W, Z X$ and $P O$ revised the article.

\section{Funding}

This study was supported by National Program for NSFC (81774017 81803959, 81973624), the Natural Science Foundation of Tianjin City (19JCYBJC28200, 18JCQNJC83300), Scientific Research Project of Tianjin Education Commission (2017KJ140) and Training Program Foundation for Innovative Research Team of Higher Education in Tianjin during the 13th
Five-Year Plan Period (TD13-5050). National Program for NSFC (81774017), Scientific Research Project of Tianjin Education Commission (2017KJ140) and Natural Science Foundation of Tianjin City (19JCYBJC28200, 18JCQNJC83300) have supported the Network Pharmacology analysis and in vitro experiment. National Program for NSFC $(81803959,81973624)$ and Training Program Foundation for Innovative Research Team of Higher Education in Tianjin during the 13th Five-Year Plan Period (TD13-5050) have supported the in vivo experiment.

\section{Availability of data and materials}

We have presented all our main data in the excel sheet in additional file.

\section{Ethics approval and consent to participate}

All animal experiments were carried out according to the National Institute of Health Guide for the Care and Use of Laboratory Animals, and were approved by the Animal Care and Use Committee of Tianjin University of Traditional Chinese Medicine (Tian, China; permit no. TCM-LAEC2019028).

\section{Consent for publication}

Not applicable.

\section{Competing interests}

The authors declare that they have no competing interests.

\section{Author details}

${ }^{1}$ Institute of Traditional Chinese Medicine, Tianjin University of Traditional Chinese Medicine, Tianjin 301617, China. ${ }^{2}$ Key Laboratory of Pharmacology of Traditional Chinese Medical Formulae, Ministry of Education, Tianjin University of Traditional Chinese Medicine, Tianjin 301617, China. ${ }^{3}$ Tianjin Medical University Cancer Hospital, Tianjin 300060, China.

Received: 12 November 2019 Accepted: 26 March 2020

Published online: 15 April 2020

\section{References}

1. Takemura G, Fujiwara H. Doxorubicin-induced cardiomyopathy: from the cardiotoxic mechanisms to management. Prog Cardiovasc Dis. 2007;49: 330-52.

2. Salvatorelli E, Menna P, Cantalupo E, Chello M, Covino E, Wolf F, et al. The concomitant management of cancer therapy and cardiac therapy. Biochim Biophys Acta. 2015;1848:2727-37

3. Lotrionte M, Biondi-Zoccai G, Abbate A, Lanzetta G, D'Ascenzo F, Malavasi V, et al. Review and meta-analysis of incidence and clinical predictors of anthracycline cardiotoxicity. Am J Cardiol. 2013;112:1980-4.

4. Tahover E, Segal A, Isacson R, Rosengarten O, Grenader T, Gips M, et al. Dexrazoxane added to doxorubicin-based adjuvant chemotherapy of breast cancer: a retrospective cohort study with a comparative analysis of toxicity and survival. Anti-Cancer Drugs. 2017;28:787-94.

5. Wu L, Wang Y, Nie J, Fan X, Cheng Y. A network pharmacology approach to evaluating the efficacy of chinese medicine using genome-wide transcriptional expression data. Evid Based Complement Alternat Med. 2013; 2013:915343

6. Shi L, Xie Y, Liao $X$, Chai $Y$, Luo $Y$. Shenmai injection as an adjuvant treatment for chronic cor pulmonale heart failure: a systematic review and meta-analysis of randomized controlled trials. BMC Complement Altern Med. 2015;15:418.

7. Jiao H, Wang XL, Chen YJ, Xiang LH, Zhang SN. Effects of Shenmai injection on afterdepolarization and triggered activities in left ventricular papillary muscle in rat cardiac hypertrophy. China J Chin Mater Med. 2014;39:2956-9.

8. Yan Z, Yuan GH, Fang P. Effect of shenmai injection on vascular endothelial dependence diastolic function in coronary heart disease patients. Chin J Integr Tradit West Med. 2017;37:302-5.

9. Jiang CZ, Ma TX, Zhao YL, Xiao JR. Observation of the therapeutic effect of Shenmai injection on doxorubicin-induced cardiotoxicity. Qilu J Oncol. 1999;6:314.

10. Liu Q, Zhang XF, Cheng XF, Wang W, Zhao HD. Clinical observation of cardiac toxicity induced by FAC regimen in female breast cancer treated by Shenmai injection. China J Chin Med. 2014;29:1090-4.

11. Yu W, Guo ZQ, Xie RX, Fan F. Clinical observation of the reduction of anthracycline-induced cardiotoxicity in patients with breast cancer by 
dexrazoxane combined with Shenmai injection. China Pharm. 2015;26: 4562-4.

12. Fang $G Y$, Zhang $Q M$, Wei $A Q$, Zhou $X M$. Shenmai injection combined with dexrazoxane in the treatment of anthracycline-induced cardiotoxicity in 31 patients with breast cancer. Chin J Crit Care Med (Electronic Edition). 2017; 10:406-7.

13. Chen $L, M a L, L u Z H$, Zhang WH. Protection effect of Shenmai injection on the myocardium injury induced by adriamycin in rats. Pract J Cancer. 2003; 18:129-31

14. Wang HS, Ma P. Effect of Shenmai injection on lipid peroxidation and calcium overloading in toxic cardiomyocytes induced by adriamycin. $J$ Anhui Tradit Chin Med Coll. 2001;20:38-40.

15. Liu XJ, Liu Y, Zhong CX, Hu CH. Protective effect of Shenmai injection on myocardial cells injured by adriamycin. China Healthcare Front. 2009;4:7-8.

16. Lv M, Yan CL, Liu HX, Wang TY, Shi XH, Liu JP, et al. Network pharmacology exploration reveals endothelial inflammation as a common mechanism for stroke and coronary artery disease treatment of Danhong injection. Sci Rep. 2017;7:15427.

17. Liu L, Du B, Zhang H, Guo X, Zhou Z, Xiu A, et al. A network pharmacology approach to explore the mechanisms of Erxian decoction in polycystic ovary syndrome. Chin Med. 2018;13:46.

18. Ru J, Li P, Wang J, Zhou W, Li B, Huang C, et al. TCMSP: a database of systems pharmacology for drug discovery from herbal medicines. J Cheminform. 2014;6:13.

19. Liu Z, Guo F, Wang Y, Li C, Zhang X, Li H, et al. BATMAN-TCM: a bioinformatics analysis tool for molecular mechANism of traditional Chinese medicine. Sci Rep. 2016;6:21146.

20. Gao L, Wang XD, Niu YY, Duan DD, Yang X, Hao J, et al. Molecular targets of Chinese herbs: a clinical study of hepatoma based on network pharmacology. Sci Rep. 2016;6:24944.

21. Ye H, Ye L, Kang H, Zhang D, Tao L, Tang K, et al. HIT: linking herbal active ingredients to targets. Nucleic Acids Res. 2011;39:1055-9.

22. Bateman A, Martin MJ, O'Donovan C, Magrane M, Apweiler R, Alpi E, et al. UniProt: a hub for protein information. Nucleic Acids Res. 2015:43:204-12.

23. Wishart DS, Knox C, Guo AC, Cheng D, Shrivastava S, Tzur D, et al. DrugBank: a knowledgebase for drugs, drug actions and drug targets. Nucleic Acids Res. 2008:36:901-6.

24. Piñero J, Queralt-Rosinach N, Bravo À, Deu-Pons J, Bauer-Mehren A, Baron $M$, et al. DisGeNET: a discovery platform for the dynamical exploration of human diseases and their genes. 2015.

25. Amberger JS, Bocchini CA, Schiettecatte F, Scott AF, Hamosh A. OMIM.org: online mendelian inheritance in man (OMIM(R)), an online catalog of human genes and genetic disorders. Nucleic Acids Res. 2015;43:789-98.

26. Shannon P, Markiel A, Ozier O, Baliqa NS, Wang JT, Ramage D, et al. Cytoscape: a software environment for integrated models of biomolecular interaction networks. Genome Res. 2003;13:2498-504.

27. Kramer A, Green J, Pollard J, Tugendreich S. Causal analysis approaches in ingenuity pathway analysis. Bioinformatics. 2014;30:523-30.

28. Li L, Zhou YF, Li YL, Wang LL, Arai H, Qi Y, et al. Aqueous extract of cortex Dictamni protects $\mathrm{H} 9 \mathrm{C} 2$ cardiomyocytes from hypoxia/reoxygenationinduced oxidative stress and apoptosis by PI3K/Akt signaling pathway. Biomed Pharmacother. 2017;89:233-44.

29. Sahu R, Dua TK, Das S, De Feo V, Dewanjee S. Wheat phenolics suppress doxorubicin-induced cardiotoxicity via inhibition of oxidative stress, MAP kinase activation, NF-KB pathway, PI3K/Akt/mTOR impairment, and cardiac apoptosis. Food Chem Toxicol. 2019;125:503-19.

30. Liu MH, Zhang Y, He J, Tan TP, Wu SJ, Guo DM, et al. Hydrogen sulfide protects $\mathrm{H} 9 \mathrm{c} 2$ cardiac cells against doxorubicin-induced cytotoxicity through the PI3K/Akt/FoxO3a pathway. Int J Mol Med. 2016;37(6):1661-8.

31. Zhao J, Yang J, Tian S, Zhang WD. A survey of web resources and tools for the study of TCM network pharmacology. Quant Biol. 2019;7(1):17-29.

32. Zhang GB, Li QY, Chen QL, Su SB. Network pharmacology: a new approach for chinese herbal medicine research. Evid Based Complement Alternat Med. 2013:2013:621423.

33. Li S, Zhang B. Traditional Chinese medicine network pharmacology: theory, methodology and application. Chin J Nat Med. 2013;11:110-20.

34. Luo TT, Lu Y, Yan SK, Xiao X, Rong XL, Guo J. Network pharmacology in research of Chinese medicine formula: methodology, application and prospective. Chin J Integr Med. 2017;23(8):635-40.
35. Fujio $Y$, Nguyen T, Wencker D, Kitsis R, Walsh K. Akt promotes survival of cardiomyocytes in vitro and protects against ischemia-reperfusion injury in mouse heart. Circulation. 2000;101:660-7.

36. Park JE, Kang YJ, Park MK, Lee YS, Kim HJ, Seo HG, et al. Enantiomers of higenamine inhibit LPS-induced iNOS in a macrophage cell line and improve the survival of mice with experimental endotoxemia. Int Immunopharmacol. 2006;6:226-33.

37. Wu W, Lee W, Wu YY, Chen D, Liu TJ, Jang A, et al. Expression of constitutively active phosphatidylinositol 3-kinase inhibits activation of caspase 3 and apoptosis of cardiac muscle cells. J Biol Chem. 2000;275: 40113-9.

38. Shiojima I, Schiekofer S, Schneider JG, Belisle K, Sato K, Andrassy M, et al. Short-term akt activation in cardiac muscle cells improves contractile function in failing hearts. Am J Pathol. 2012;181:1969-76.

39. Huang $X$, Wang Y, Wang Y, Yang L, Wang J, Gao Y. Ophiopogonin D reduces myocardial ischemia-reperfusion injury via upregulating CYP2J3/ EETs in rats. Cell Physiol Biochem. 2018;49:1646-58.

40. Wang X, Chen L, Wang T, Jiang X, Zhang H, Li P, et al. Ginsenoside Rg3 antagonizes adriamycin-induced cardiotoxicity by improving endothelial dysfunction from oxidative stress via upregulating the Nrf2-ARE pathway through the activation of akt. Phytomedicine. 2015;22:875-84.

41. Li YH, Li YY, Fan GW, Yu JH, Duan ZZ, Wang LY, et al. Cardioprotection of ginsenoside Rb1 against ischemia/reperfusion injury is associated with mitochondrial permeability transition pore opening inhibition. Chin J Integr Med. 2016:1-10

42. Chen $Y$, Tang $Y$, Zhang $Y$, Huang $X$, Xie $Y$, Xiang $Y$. A metabolomic study of rats with doxorubicin-induced cardiomyopathy and Shengmai injection treatment. Plos One. 2015;10(5):e0125209.

43. Shah $\mathrm{H}$, Haridas N. A serial follow up study of cardiac marker enzymes during the week after acute myocardial infarction. Indian J Clin Biochem. 2007;22(1):33-6.

44. Wei S, Mao L, Liu B, Zhong L. Serum biomarkers and the prognosis of AMI patients; 2013.

\section{Publisher's Note}

Springer Nature remains neutral with regard to jurisdictional claims in published maps and institutional affiliations.

\section{Ready to submit your research? Choose BMC and benefit from:}

- fast, convenient online submission

- thorough peer review by experienced researchers in your field

- rapid publication on acceptance

- support for research data, including large and complex data types

- gold Open Access which fosters wider collaboration and increased citations

- maximum visibility for your research: over $100 \mathrm{M}$ website views per year

At $\mathrm{BMC}$, research is always in progress.

Learn more biomedcentral.com/submission 